
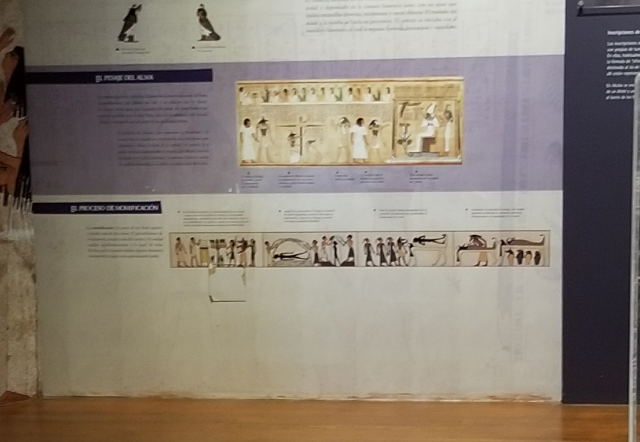
플

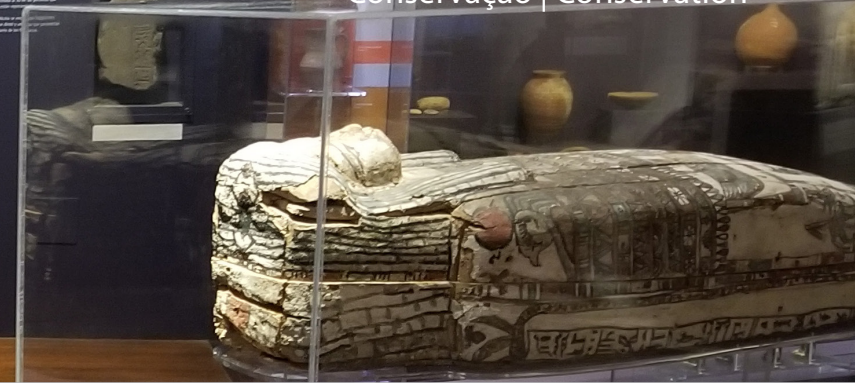

\title{
Análisis de la carga fúngica en el aire de la sala "Fragmentos de Historia a Orillas del Nilo" y del exterior del Museo de La Plata, Argentina
}

\author{
Andrea C. Mallo, Daniela S. Nitiu*, Lorena A. Elíades, Mauro García Santa Cruz, Mario C. N. \\ Saparrat \\ * co-primer autor
}

Resumen: Se realizó un estudio aerobiológico de esporas fúngicas en el aire, su dinámica estacional y el análisis de la incidencia de los factores ambientales en el riesgo de biodeterioro en la sala Egipcia del Museo de La Plata. Se detectaron 18 tipos de unidades fúngicas de dispersión (esporas y otros elementos vegetativos) en la carga microrobiana. El promedio anual de UFC $/ \mathrm{m}^{3}$ aire para los 3 sitios de la sala mostró 31158,90 elementos, comparado con 28046 en el sitio exterior. Aspergillus niger, Beauveria, Fusarium oxysporum y Penicillium fueron los tipos más abundantes. El análisis de la variación diaria de temperatura y humedad en el interior de la Sala, indicó que la variable fuera de rango fue la humedad relativa. Las estrategias combinadas de monitoreo aerobiológico proporcionan nuevas herramientas para la evaluación y predicción del tiempo de vida de los diferentes materiales.

Palabras clave: Sala egipcia, Museo de La Plata, aeromicobiota, carga fúngica, biodeterioro, parámetros ambientales

\section{Analysis of the fungal load in the air of the hall "Fragments of history at the shores of the Nile" and of the outside of the La Plata Museum, Argentina}

Abstract: An aeromicological study of fungal spores in the air, its seasonal dynamics and the analysis of the incidence of environmental factors on the risk of biodeterioration were carried out in the Egyptian room of the Museum of La Plata. Eighteen types of fungal propagules were detected. The annual average of UFC $/ \mathrm{m}^{3}$ air for the 3 sites of the room showed 31158.90 elements, compared to 28046 in the external site. Aspergillus niger, Beauveria, Fusarium oxysporum and Penicillium were the most abundant types. The analysis of the daily variation of temperature and humidity inside the room indicated that the out of range variable was relative humidity. The combined strategies of aerobiological monitoring provide new tools for the evaluation and prediction of the life time of the different materials.

Keyword: Egiptian Hall, La Plata Museum, aeromicobiota, fungal load, biodeterioration, environmental parameters

\section{Análise da carga fúngica no ar da sala "Fragmentos da história das margens do Nilo" e do exterior do Museu de La Plata, Argentina}

Resumo: Foi realizado um estudo aerobiológico dos esporos de fungos no ar, a sua dinâmica sazonal e a análise da incidência de fatores ambientais no risco de deterioração biológica na sala egípcia do Museu de La Plata. Foram detetados 18 tipos de unidades de dispersão fúngica (esporos e outros elementos vegetativos) na carga microbiana. A média anual de UFC/ $\mathrm{m}^{3}$ de ar para os três locais da sala apresentou 31.158,90 elementos, em comparação com 28046 no local externo. Aspergillus niger, Beauveria, Fusarium oxysporum e Penicillium foram os tipos mais abundantes. A análise da variação diária de temperatura e humidade no interior da sala indicou que a variável fora do intervalo foi a humidade relativa. As estratégias combinadas de monitoração aerobiológico proporcionam novas ferramentas para a avaliação e previsão do tempo de vida de diferentes materiais.

Palavras-chave: Sala egípcia, Museu de La Plata, aeromicrobiota, carga fúngica, biodeterioração, parâmetros ambientais 


\section{Introducción}

La presencia de esporas y otros tipos de elementos fúngicos en el aire interior de museos y otros sitios donde se custodian colecciones de piezas de importancia histórica y su potencial germinación sobre materiales asociados, constituye una amenaza para la conservación del patrimonio natural y cultural (Harvey 1977). Ello se debe a la variedad de sustratos que lo componen y a la capacidad degradadora que poseen los hongos (Sterflinger \& Piñar 2013). La contaminación aeromicológica impacta negativamente en las colecciones que se custodian en los museos afectando la conservación de los ejemplares, soportes, la calidad del aire así como la salud del personal y los visitantes. La mayor parte de las colecciones que se preservan y/o exhiben en los museos son de naturaleza orgánica (Sterflinger \& Piñar 2013), caracterizándose incluso por su higroscopicidad, esto es, la alta capacidad de retención de agua, siendo a la vez una fuente secundaria de inóculo fúngico. A la vez ello puede implicar que un significativo incremento del contenido de humedad en el material y su soporte, especialmente cuando los objetos están sometidos a una ventilación insuficiente y a una humedad relativa superior al $65 \%$, pueden tornarlos más vulnerables al biodeterioro. Además, el fenómeno de "subtropicalización" del clima provocado por el Cambio Climático ha modificado el régimen de lluvias en la región incidiendo en los factores críticos de temperatura y humedad que condicionan la alteración de los materiales por la actividad biológica (Gómez 2009).

El Museo de Ciencias Naturales de La Plata, Argentina $\left(34^{\circ} 55^{\prime} \mathrm{S}, 57^{\circ} 57^{\prime} \mathrm{O}\right)$, dispone de una sala denominada "Fragmentos de historia a orillas del Nilo", un recinto que alberga una exposición permanente que presenta fragmentos de arenisca que pertenecieron al Complejo de Aksha ubicado a orillas del río Nilo en la frontera entre Sudán y Egipto. Específicamente se trata del resguardo de parte de las piezas egipcias que fueron donadas por Dardo Rocha, en el siglo XIX y la colección rescatada por una expedición arqueológica franco-argentina subsidiada por UNESCO con motivo de la construcción de la represa de Aswan Sadd elAli entre los años 1960 y 1970. Se trata del templo egipcio Aksha, cementerios de diferentes culturas nubias, una tumba en Beidir y otro templo construido por los pueblos prehistóricos. Las piezas conservan inscripciones jeroglíficas cuya interpretación, junto a otras evidencias, permite recuperar diversos aspectos de la vida en el antiguo Egipto (http://www.museo.fcnym.unlp.edu.ar egipcia). Aunque esta colección está bien conservada en vitrinas especiales en la sala, recientemente reinaugurada en el 2013 luego de un intenso trabajo de restauración y puesta en valor en coordinación con la Unidad de Conservación y Exhibición del Museo de La Plata, no hay información disponible sobre la carga fúngica del aire interior de la sala y su variación estacional.

El objetivo de este trabajo fue proporcionar información acerca de la calidad del aire interior de la Sala "Fragmentos de Historia a Orillas del Nilo" instalada en el Museo de Ciencias Naturales de La Plata, Argentina, en relación con la carga fúngica en el aire, su dinámica estacional y el análisis de los factores microambientales que son claves en el riesgo potencial de biodeterioro de las piezas en exposición.

\section{Materiales y métodos}

\section{- Sitio de estudio}

La sala posee una superficie de $300 \mathrm{~m}^{2}$ y se encuentra en la planta alta del Museo [figura 1]. Se seleccionaron 3 áreas representativas: sitio 1: correspondiente a un área que expone más de 300 piezas que incluyen bloques de arenisca pertenecientes a dinteles, frisos, jambas de puertas con inscripciones de jeroglíficos, cerámicas y dos ataúdes con sus respectivas momias; sitio 2: un área que tiene una puesta museográfica que incluye una maqueta del complejo y otra del templo de Aksha, incluyendo un libro digital que relata el hallazgo y las distintas expediciones que se sucedieron desde el siglo pasado y ficheros interactivos que amplían la información sobre el Nilo y la escritura egipcia; sitio 3: zona de la sala que exhibe cerámicas que pertenecieron a cementerios de otras culturas de la misma región egipcia, como una gárgola meroítica y un capitel de la época cristiana; y sitio 4: entorno exterior del Museo con una variada vegetación implantada [figura 2].

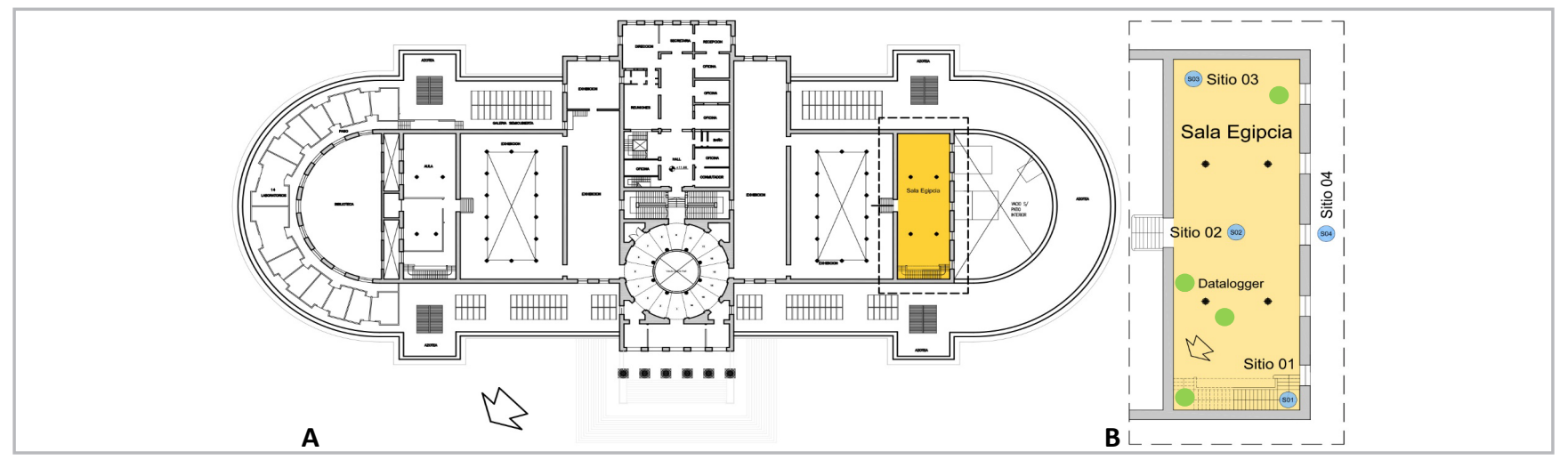

Figura 1.- A: Plano del Museo de Ciencias Naturales, planta alta, localización de la sala "Fragmentos de Historia a Orillas del Nilo" (amarillo). B: detalle de la ubicación de los dataloggers (verde) y puntos de muestreo microbiológico (azul). 


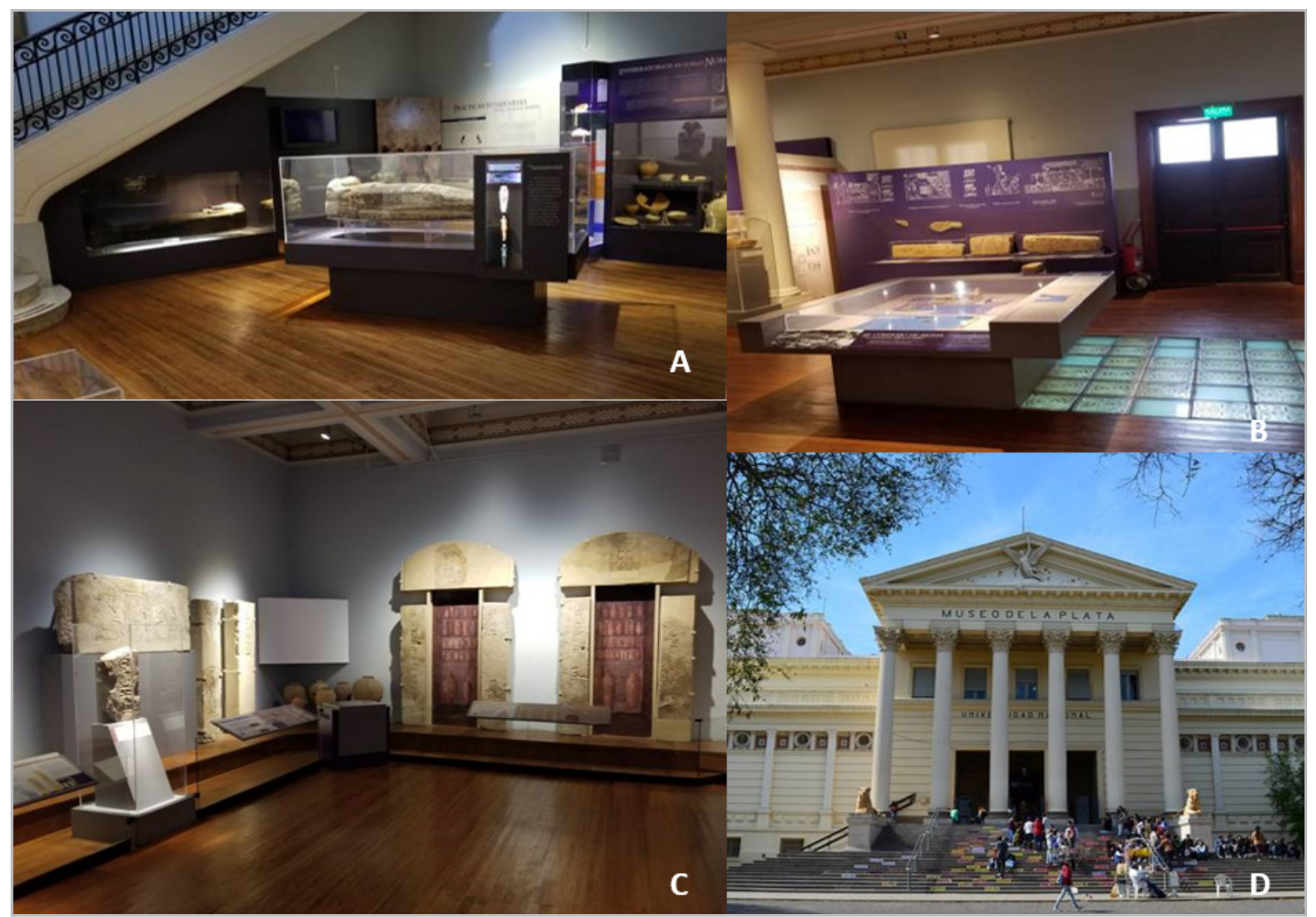

Figura 2.- Vista general de los sitios estudiados. A- Sitio 1 (ala Norte); B- Sitio 2 (zona ingreso), C- Sitio 3 (ala Sur), D- Sitio 4 (exterior)

\section{—Muestreo micológico del aire}

Se llevó a cabo el muestreo del aire asociado a los 4 sitios indicados 2 veces en cada estación del año desde mes de julio del 2015 (invierno y primavera), hasta junio de 2016 (verano y otoño) a las 9:30 de la mañana, utilizando un método volumétrico con un muestreador tipo HirstZefon Z-Lite IAQ Air SamplingPump ${ }^{\oplus}$. Esta metodología se llevó a cabo basada en el principio de impacto inercial de las partículas y adaptado para el posterior cultivo de las muestras sobre un medio estándar para el crecimiento de hongos ambientales.

1- Se tomaron muestras por triplicado en cada sitio, utilizando portafiltros Millipore equipados con papel de filtro estéril. El sistema resultante se aplicó a la bomba de succión de aire durante 5 minutos a una velocidad de 0,015 $\mathrm{m}^{3}$ por minuto.

2-En el laboratorio, cada filtro se trituró y se homogeneizó en $20 \mathrm{ml}$ de agua estéril bajo condiciones asépticas, obteniendo una suspensión que se utilizó como fuente de inóculo.

3- Un mililitro de alícuotas decimales seriadas de cada suspensión de partida se sembró en placas conteniendo un medio agarizado con Rosa de Bengala x \% (Biopack, C. I.45440 articulo 947.901; Miller, et al. 2000) suplementado con sulfato de estreptomicina $(0,5 \%)$ y cloramfenicol $(0,25 \%)$. Se realizaron tres réplicas de siembra por dilución de la suspensión analizada.

4- Las placas se incubaron a $25^{\circ} \mathrm{Cy} 63 \%$ de humedad relativa en la oscuridad hasta desarrollo de colonias, las cuales fueron identificadas taxonómicamente y cuantificadas como unidades formadoras de colonias (CFU) y convertidas en unidades volumétricas (Baxter 2006).

5- La identificación de los géneros y especies fúngicas se realizó sobre la base de características morfológicas y culturales, utilizando bibliografía de referencia (Barron 1968; Carmichael et al. 1980; Domsch et al. 1993; Ellis 1971, 1976).

\section{- Análisis de datos}

Se calculó la densidad relativa (RD) de los géneros fúngicos en las muestras según Smith (1980) utilizando la siguiente fórmula:

Número de colonias de un determinado taxa

$\mathrm{RD}(\%)=\frac{\text { en una muestra }}{\text { Número total de colonias fúngicas contabilizadas }}$ en la muestra 
Donde taxa alude a las distintas categorías taxonómicas (especie, género o familia) que se pudieron determinar en el cultivo

Se estimó la frecuencia relativa (FR) de cada género fúngico de acuerdo a Esquivel (2003), que distingue 5 categorías: abundante (81 - $100 \%)$; común (61 - 80\%); frecuente (41$60 \%)$; ocasional $(21-40 \%)$ y raro $(0,1-20 \%)$; (Borrego \& Perdomo 2016), empleando la fórmula:

$$
\text { FR }(\%)=\frac{\begin{array}{l}
\text { Número de veces que un género es detectado } \\
\text { en sitio }
\end{array}}{\text { Número de veces que el género es detectado en }}
$$

Se utilizó el cociente de similitud de Sørensen (1957) (QS) para comparar la similitud de la composición de géneros fúngicos de dos muestras según la siguiente fórmula:

$\mathrm{QS}=\frac{2 \mathrm{a}}{2 \mathrm{a}+\mathrm{b}+\mathrm{c}}$

Donde a es el número de géneros comunes en ambas muestras; $b$ el número de géneros hallados solo en una muestra y c el número de géneros detectados solo en la otra muestra.

Para detectar las diferencias significativas en las comparaciones de las concentraciones fúngicas entre los sitios y entre estaciones, se utilizó el test de Student, previa comprobación de la bondad de ajuste de dos distribuciones mediante el test de Kolmogorov - Smirnov.

También se calculó el índice interior/exterior (I/O) siguiendo a Levetin et al. (1995) que considera que cuando la concentración de microorganismos en el aire interior es similar al aire exterior y el índice Indoor/Outdoor es inferior a 1,5; el ambiente puede considerarse apropiado.

\section{—Evaluación de los parámetros ambientales}

Para la evaluación del edificio se utilizó la metodología desarrollada por el Laboratorio de Arquitectura y Hábitat Sustentable de la FAyU, UNLP en el Proyecto“Evaluación de las necesidades de control del entorno para la conservación de los museos de la Red de Museos de la UNLP. Adecuación del procedimiento del Getty Conservation Institute (GCl)". En este proyecto se adaptó a nuestra región la metodología publicada por el $\mathrm{GCl}$ en el documento "Modelo propuesto para evaluar las necesidades de control del entorno museístico" (Avrami et al. 1999).

Se estimaron los valores de temperatura y humedad relativa de diferentes puntos en cada sitio utilizando microadquisidores de datos (dataloggers) durante todo el periodo del monitoreo (desde el 1 de julio del 2015 hasta junio del 2016) con una frecuencia de 10 minutos (García Santa Cruz \& Gómez 2014). Siguiendo la Norma UNI10829:1999 (Wills et al., 2014) [figura 1b]. Los datos registrados fueron comparados con los valores de referencia recomendados para la conservación de diferentes objetos ubicados en el sitio de análisis de la sala (correspondientes a las categorías "momias", "madera pintada", "cerámica", y "piedras"), que están representados dentro de un rango de tolerancia y que son variables según la composición química de los mismos [tabla 3].

\section{Resultados}

La tabla 1 muestra los tipos fúngicos identificados cuando diferentes muestras de aire de los 4 sitios se utilizaron como inóculo fúngico sobre un medio agarizado correspondientes a las 8 campañas evaluadas. Aunque se distribuyeron varias diluciones decimales de hasta 1: 10 en placas de agar, solo se obtuvieron datos informativos en el muestreo de su propio filtro y la suspensión no diluida ( $\leq 40$ UFC por placa). Durante el período total monitoreado se detectaron 18 tipos fúngicos, 17 de los cuales pertenecen al phylum Ascomycota y uno al phylum Basidiomycota (Rodothorula F. C. Harrison). Nueve de ellos fueron identificados hasta el nivel genérico mientras que los 9 restantes fueron determinados a nivel específico. Adicionalmente se recuperaron dos tipos de elementos fúngicos vegetativos que solo diferenciaron un "micelio hialino"y un"micelio dematiáceo" cuando fueron incubados en el medio agarizado. La figura 3 muestra la frecuencia de aparición de los tipos fúngicos hallados en el aire de los 4 sitios estudiados. Con excepción de Acremonium Link., Alternaria Nees., Fusarium solani (Mart.) Sacc. y Trichoderma Pers., los tipos fúngicos identificados se detectaron en las 8 campañas, aunque revelaron distinta frecuencia. El promedio anual de UFC/m3 aire de carga fúngica para los 3 sitios de la sala mostró 31158,90 elementos, comparado con 28046 en el sitio exterior. El análisis del índice RD estimado para los tipos predominantes en todo el período en los 4 sitios indica que Aspergillus niger Tiegh. representó el 12,94\% de la concentración total, Beauveria Vuill. el 21,23 $\%$, Fusarium oxysporum Schltdlel. 13,77 \% y Penicillium Link el $17,64 \%$.

Analizando cada sitio de la sala, en el sitio 1 se registró un total anual de $37607 \mathrm{UFC} / \mathrm{m}^{3}$ aire con un promedio mensual de 4700,85 ( $\pm 3283,83$ ), con la mayor concentración en el primer muestreo de invierno (11810 UFC/m $\mathrm{m}^{3}$ aire) y la menor en el primero de verano (2220 UFC/ $\mathrm{m}^{3}$ aire) [figura 4]. La aeromicobiota de este sitio estuvo representada por 16 tipos, con Penicillium sp. como dominante (11396 UFC/ m3 aire) y una densidad relativa de 30,3\%; [figura 5 a].

En el sitio 2 se registró un total anual de $28652 \mathrm{UFC} / \mathrm{m}^{3}$ aire con un promedio mensual de 3581,60 ( $\pm 1978,41$ ); la mayor concentración se registró en el primer muestreo de invierno (7104 UFC/m³ aire) y la muestra de febrero registró 
Tabla 1.- Géneros y especies fúngicas recuperadas en los sitios según el momento de muestreo.

\begin{tabular}{|c|c|c|c|c|c|}
\hline Tipo fúngico & Phylum & Invierno & Primavera & Verano & Otoño \\
\hline Acremonium sp. & Ascomycota & & & & $1,2,3$ \\
\hline Alternaria sp. & Ascomycota & 3,4 & 3 & & \\
\hline Aspergillus sp. & Ascomycota & & 2,3 & $1,2,3,4$ & \\
\hline Aspergillus niger & Ascomycota & $1,2,3,4$ & $1,2,3,4$ & 4 & $1,2,4$ \\
\hline Aspergillus terreus & Ascomycota & $1,3,4$ & & & $1,3,4$ \\
\hline Beauveria sp. & Ascomycota & $1,2,4$ & & & $1,2,4$ \\
\hline Cladosporium sp. & Ascomycota & 1,2 & 2 & 2 & 2 \\
\hline Cladosporium cladosporioides & Ascomycota & $1,3,4$ & 3 & & $1,3,4$ \\
\hline Cladosporium herbarum & Ascomycota & 4 & & & \\
\hline Fusarium oxisporum & Ascomycota & 2,3 & $1,2,3,4$ & $1,2,3$ & $1,2,3,4$ \\
\hline Fusarium solanii & Ascomycota & 2 & & & 1 \\
\hline Micelio blanco & Indeterminado & 1 & & & \\
\hline Micelio dematiaceo estéril & Indeterminado & 1 & $1,2,3$ & 2,3 & $1,2,3$ \\
\hline Paecilomyces lilacinus & Ascomycota & 1,3 & & & $1,2,3$ \\
\hline Penicillium sp. & Ascomycota & $1,2,3$ & $1,2,3$ & 1 & $1,2,3$ \\
\hline Penicillium restrictum & Ascomycota & 4 & & & \\
\hline Penicillium rubrum & Ascomycota & 1,2 & & 1,4 & 1,4 \\
\hline Rhodotorula sp. & Basidiomycota & 1,2 & $1,2,3,4$ & $2,3,4$ & $1,2,3,4$ \\
\hline Talaromyces sp & Ascomycota & 1,3 & & 1,3 & 1,2 \\
\hline Trichoderma sp. & Ascomycota & 2,4 & & & \\
\hline
\end{tabular}

\begin{tabular}{|c|c|c|c|c|}
\hline Tipo fúngico & sitio 1 & sitio 2 & sitio 3 & sitio 4 \\
\hline \multicolumn{5}{|l|}{ Acremonium sp. } \\
\hline \multicolumn{5}{|l|}{ Alternaria sp. } \\
\hline \multicolumn{5}{|l|}{ Aspergillus sp } \\
\hline \multicolumn{5}{|l|}{ Aspergillus niger } \\
\hline \multicolumn{5}{|l|}{ Aspergillus terreus } \\
\hline \multicolumn{5}{|l|}{ Beauveria sp. } \\
\hline \multicolumn{5}{|c|}{ Cladosporium cladosporioides } \\
\hline \multicolumn{5}{|c|}{ Cladosporium herbarum } \\
\hline \multicolumn{5}{|l|}{ Fusarium oxisporum } \\
\hline \multicolumn{5}{|l|}{ Fusarium solani } \\
\hline \multicolumn{5}{|l|}{ Micelio blanco } \\
\hline \multicolumn{5}{|c|}{ Micelio dematiaceo etéril sp } \\
\hline \multicolumn{5}{|c|}{ Paecilomyces lilacinus } \\
\hline \multicolumn{5}{|l|}{ Penicillium sp. } \\
\hline \multicolumn{5}{|c|}{ Penicillium restrictum } \\
\hline \multicolumn{5}{|l|}{ Penicillium rubrum } \\
\hline \multicolumn{5}{|l|}{ Rhodotorula sp. } \\
\hline \multicolumn{5}{|l|}{ Talaromyces sp } \\
\hline \multicolumn{5}{|l|}{ Trichoderma sp. } \\
\hline \multicolumn{5}{|c|}{ Clasificación de Yadav y Madelin } \\
\hline \multicolumn{5}{|c|}{$\begin{array}{l}\text { Categoría Frecuenc ia de } \\
\text { aparic ión }\end{array}$} \\
\hline \multicolumn{5}{|l|}{ Muy común 80 - 100\% } \\
\hline \multicolumn{5}{|l|}{ Común $61-80 \%$} \\
\hline \multicolumn{5}{|l|}{ Frecuente $41-60 \%$} \\
\hline \multicolumn{5}{|l|}{ Ocasiona I $21-40 \%$} \\
\hline Raro $0,1-20 \%$ & & & & \\
\hline
\end{tabular}

Figura 3.- Frecuencia de aparición de los tipos fúngicos aislados en los distintos sitios en el total de muestras tomadas por sitio. 
el mínimo (947 UFC/m³ aire) [figura 4] La aeromicobiota estuvo representada por 15 tipos fúngicos con Beauveria predominando en todo el período (7341UFC/m³aire) y una densidad relativa de $25,62 \%$ [figura $5 \mathrm{~b}$ ].

El sitio 3 registró un total anual de $27217,2 \mathrm{UFC} / \mathrm{m}^{3}$ aire y un promedio mensual de 3402,15 ( \pm 746$)$ la mayor concentración total se obtuvo en el recuento de noviembre (4144 UFC/m³ $\mathrm{m}^{3}$ aire) y febrero registró el mínimo (2012 $\mathrm{UFC} / \mathrm{m}^{3}$ ) [figura 4]. La aeromicobiota estuvo representada por 12 tipos fúngicos siendo Fusarium oxysporum el tipo predominante con $6630 \mathrm{UFC} / \mathrm{m}^{3}$ y una densidad relativa de $24,36 \%$ [figura $5 c$ ].

El sitio 4 presentó un total anual: $28046 \mathrm{UFC} / \mathrm{m}^{3}$; con un promedio de $3509,45 \mathrm{UFC} / \mathrm{m}^{3}( \pm 3728,30)$; el mes de mayor concentración total fue junio con $12461 \mathrm{UFC} / \mathrm{m}^{3}$ aire y febrero registró el mínimo (1465 UFC/m³) [figura 4]. Se detectaron 16 tipos fúngicos, Cladosporium cladoporioides (Fresen.) G.A. de Vries fue el tipo predominante con 6985 $\mathrm{UFC} / \mathrm{m}^{3}$ aire y una densidad relativa de $24,36 \%$ [figura $5 \mathrm{~d}$ ].

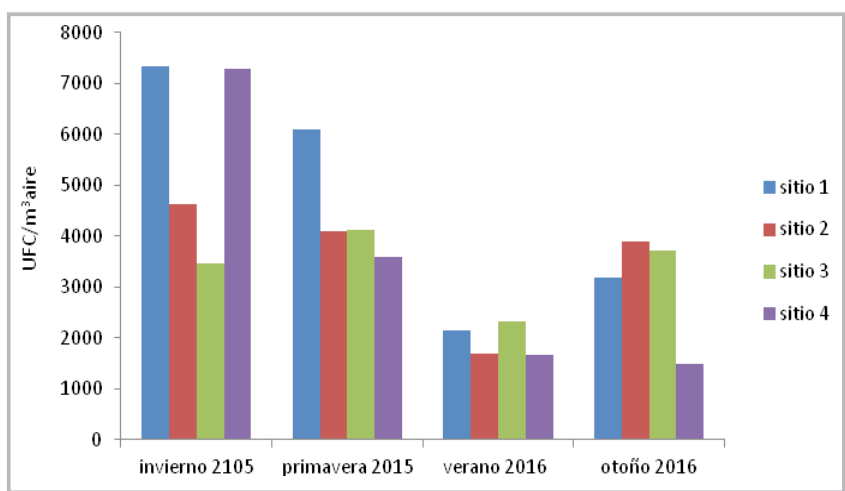

Figura 4.- Comportamiento estacional en términos de concentración total de UFC/m3 de aire en los 4 sitios estudiados. Sitio 1, ala Norte; sitio 2, zona ingreso; sitio 3, ala Sur; sitio 4, exterior.
Analizando las concentraciones registradas en las 4 estaciones en el interior de la sala, se detectaron diferencias significativas entre las muestras de invierno/verano, primavera/verano, primavera/otoño y otoño/verano ( $T$ de Student, $p<0,05$; [tabla 2]. Sin embargo, no se detectaron diferencias en ninguna de las concentraciones de las muestras correspondientes a cada sitio cuando fueron comparadas entre sí. En este sentido, el análisis de la riqueza promedio de géneros fúngicos de los sitios interiores comparado con el exterior, utilizando el índice de similitud, mostró una alta superposición y/o constante intercambio de la carga fúngica lo largo del año (QS Sitios 1-4 = 0,55; QS Sitios 2-4 =0,55; QS Sitios 3-4: 0,53). Beauveria y Rhodotorula son típicos del ambiente exterior mientras que Fusarium y Penicillium son característicos en el interior de la sala.

Cuando se aplicó el índice I/O entre los sitios 1, 2 y 3 y el ambiente exterior los resultados arrojaron valores debajo de $1,5(1,33,1,02$ y 1,05 respectivamente). Asimismo, el análisis de la densidad relativa entre los sitios 1 (ala Norte), 2 (zona de ingreso) y 3 (ala Sur) con el sitio 4 (ambiente exterior) [figura 6] evidenció un fuerte intercambio que se manifestó en la similitud en la diversidad. Los sitios interiores compartieron altos porcentajes de coincidencia en los tipos fúngicos. Se registró un $73 \%$ de coincidencia entre los sitios 1 y 4 con predominio de Aspergillus y Penicillium en el interior y Beauveria y Rhodotorula en el exterior. En el sitio 2 hubo un $60 \%$ de coincidencia en la riqueza fúngica y los tipos que predominaron fueron Aspergillus, Beauveria y Fusarium. Analizando el sitio 3 se registró un $68 \%$ de coincidencia predominando Aspergillus, Cladosporium y Fusarium. Por otra parte, el análisis de la riqueza en función de la estacionalidad [figura 7], puso en evidencia que el otoño fue la estación en la cual hubo mayor cantidad de tipos fúngicos en todos los sitios, seguida por la primavera, el invierno y la estación más pobre, el verano. [figura 7].

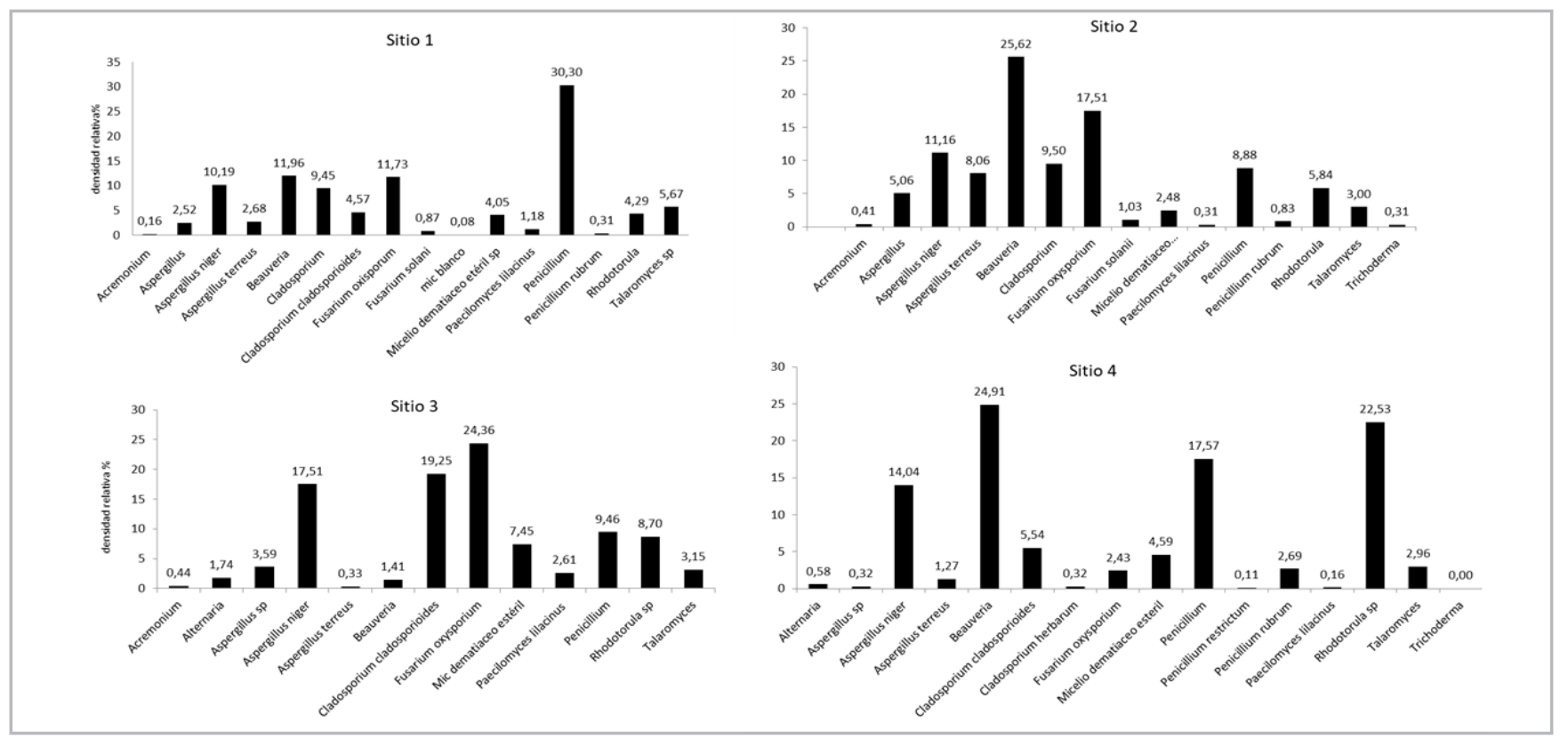

Figura 5.- Densidad relativa porcentual de los tipos fúngicos hallados en los cuatro sitios estudiados. 


\begin{tabular}{|c|c|c|c|c|c|c|c|c|}
\hline & \multicolumn{2}{|c|}{ Invierno } & \multicolumn{2}{|c|}{ Primavera } & \multicolumn{2}{c|}{ Verano } & \multicolumn{2}{c|}{ Otoño } \\
\hline & $T$ & $\mathrm{p}<0,05$ & $\mathrm{~T}$ & $\mathrm{p}<0,05$ & $\mathrm{~T}$ & $\mathrm{p}<0,05$ & $\mathrm{~T}$ & \\
\hline Invierno & & & & & & \\
\hline Primavera & 0,23 & 0,41 & & & & \\
\hline Verano & $\mathbf{2 , 0 2}$ & $\mathbf{0 , 0 4}$ & $\mathbf{5 , 1 9}$ & $\mathbf{0 , 0 0}$ & & & \\
\hline Otoño & 0,99 & 0,17 & $\mathbf{2 , 0 2}$ & $\mathbf{0 , 0 4}$ & $\mathbf{- 3 , 6 8}$ & $\mathbf{0 , 0 0}$ & \\
\hline
\end{tabular}

Tabla 2.- Análisis estadístico de las concentraciones de UFC/m3 en las 8 campañas realizadas (en negrita, diferencias significativas).

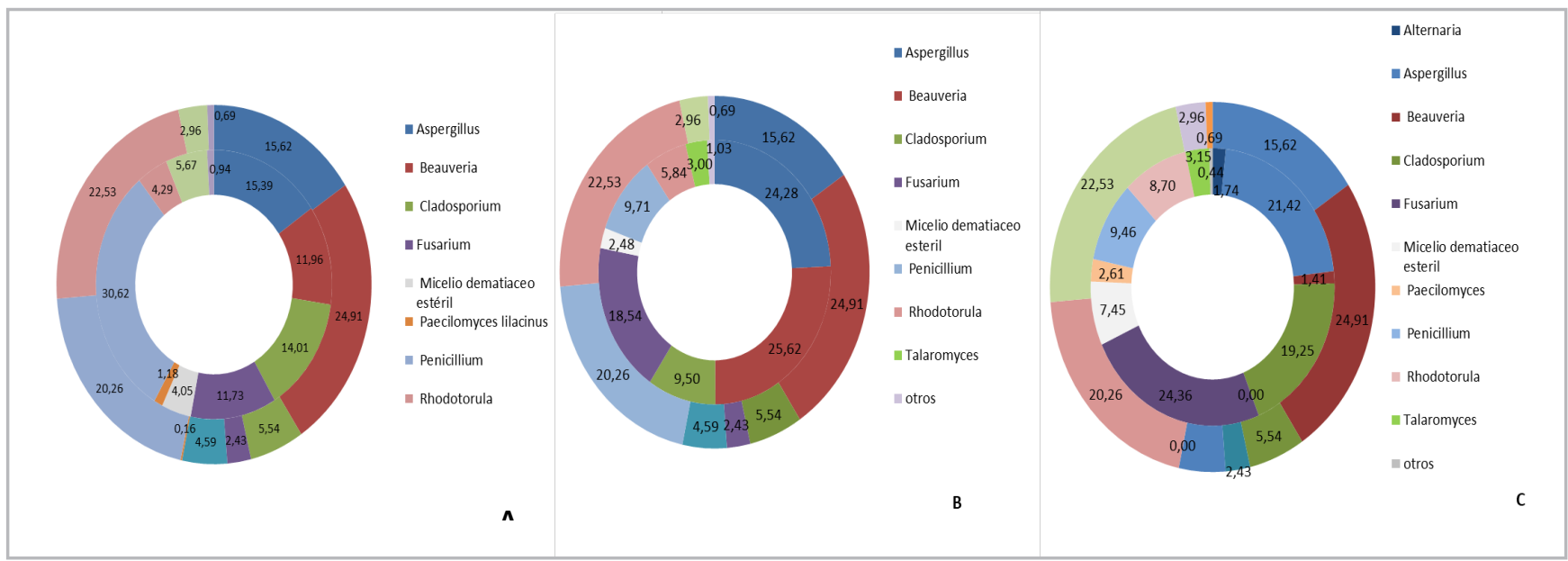

Figura 6.- Comparación cuali-cuantitativa del espectro aerobiológico entre los sitios de la Sala Egipcia (círculos interiores de los gráficos) con el exterior (ć́rculo exterior de los gráficos). A- sitio 1 vs sitio 4; B- sitio 2 vs sitio 4; C- sitio 3 vs sitio 4.

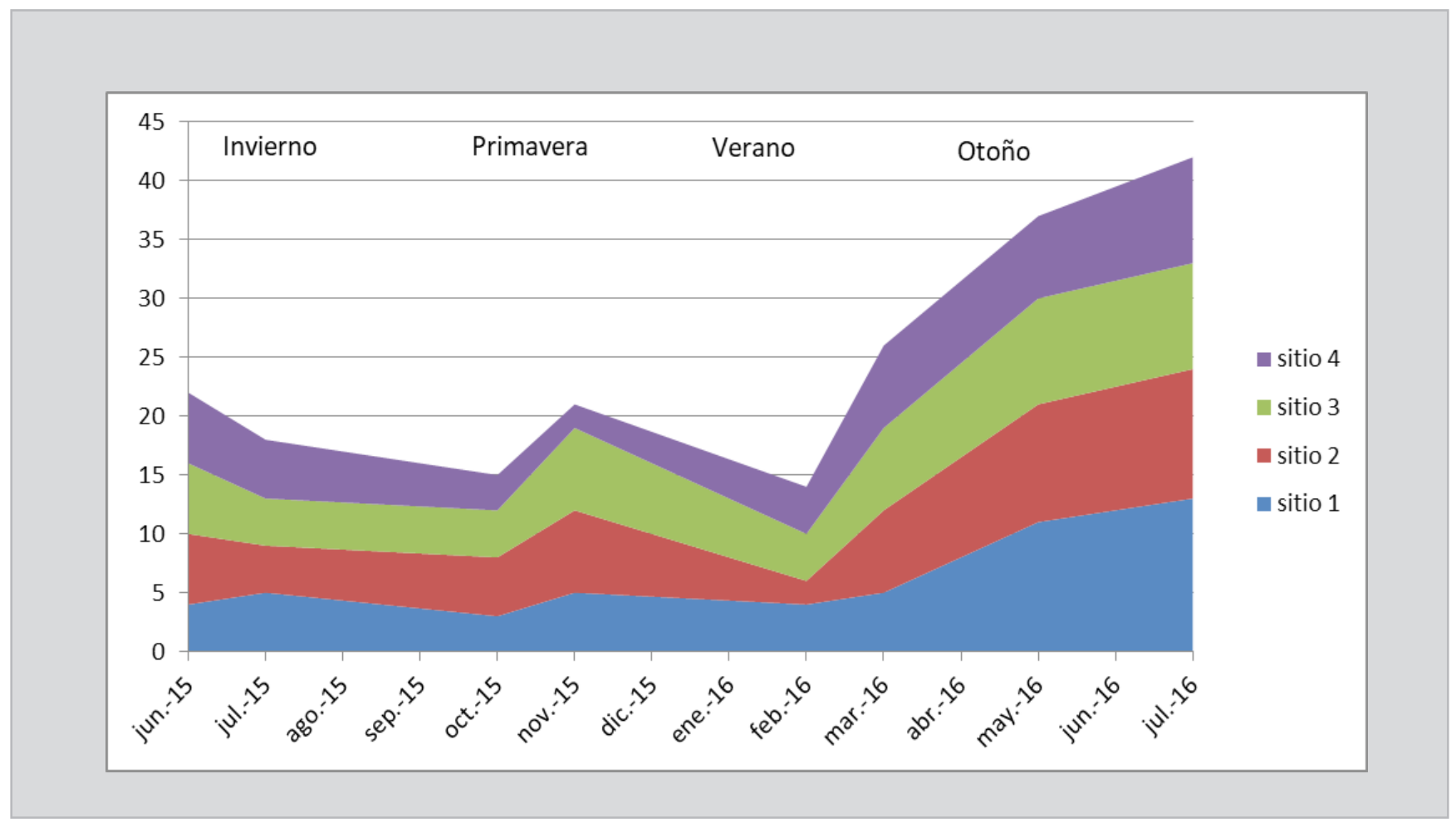

Figura 7.- Número de tipos fúngicos registrados cada estación del muestreo. 


\section{Análisis de los parámetros ambientales}

El comportamiento de los parámetros de temperatura y humedad relativa (HR) en los sitios estudiados puede analizarse a partir del histograma que se presenta en la figura 8. La temperatura se comportó de forma similar en todos los casos y la mayor dispersión se dio en los valores de humedad relativa.

Considerando las características de los objetos que alberga la Sala Egipcia y analizando la bibliografía específica se determinaron los valores de referencia recomendados para su conservación, correspondientes a las categorías "momias", "madera pintada", "cerámica", "piedras" (Norma UNI10829:1999; Wills et al. 2014). Dependiendo de las categorías, se cuenta con valores óptimos de conservación y valores de tolerancia, estos últimos constituyen un rango ampliado sobre los primeros [tabla 3]. A partir del análisis de la variación diaria de temperatura y humedad en el exterior e interior de la Sala Egipcia durante las cuatro estaciones de muestreo y la comparación con la tabla de referencia para los principales tipos de materiales que se hallan en la sala se puede verificar que la variable fuera de rango fue la humedad relativa [figura 9].

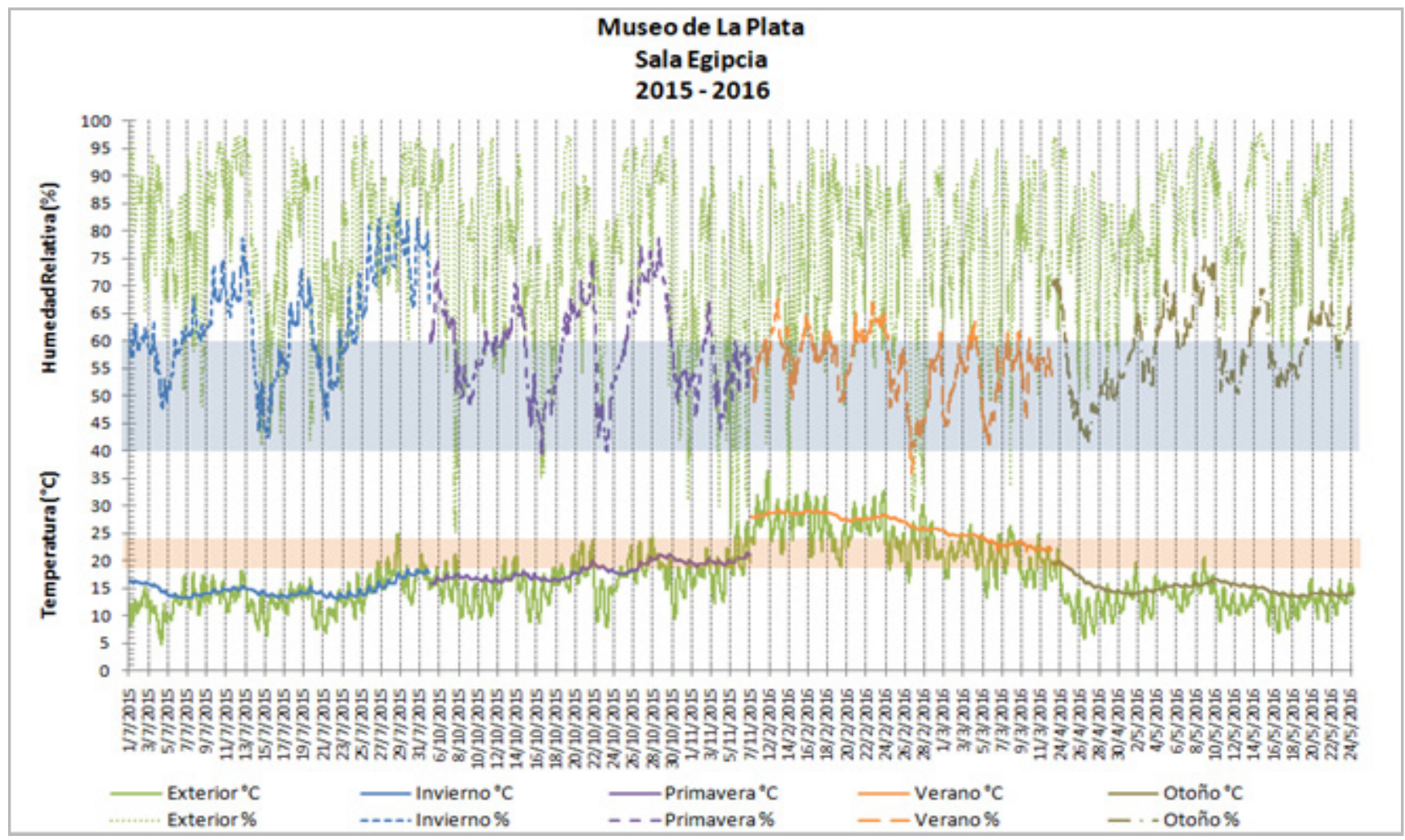

Figura 8.- Histograma de la evolución de los parámetros de temperatura y humedad relativa (HR) durante el período 2015 - 2016 en la Sala Egipcia del Museo de La Plata.

\begin{tabular}{|c|c|c|c|c|}
\hline BIENES DE INTERÉS HISTÓRICO O ARTÍSTICO & $\Theta \mathrm{T}$ & $\Delta \theta \operatorname{Tmax}$ & HRO & $\Delta \mathrm{HRmax}$ \\
\hline \multicolumn{5}{|c|}{ MATERIALES / OBJETOS DE NATURALEZA ORGÁNICA } \\
\hline Animales, órganos anatómicos disecados, momias & 21 a 23 & 1.5 & 20 a 35 & - \\
\hline Colecciones etnográficas, máscaras, cuero, indumentaria de cuero & 19 a 24 & 1.5 & 45 a 60 & 6 \\
\hline $\begin{array}{l}\text { Esculturas de madera policroma, madera pintada, pintura sobre } \\
\text { madera, iconos }\end{array}$ & 19 a 24 & 1.5 & 50 a 60 & 4 \\
\hline \multicolumn{5}{|c|}{ MATERIALES DE NATURALEZA INORGÁNICA } \\
\hline Porcelana, cerámica, gres, terracota, tejas, tejas de extracción & NR & - & NR & 10 \\
\hline Piedras, rocas, minerales, meteoritos (porosos) estables & 19 а 24 & - & 40 a 60 & 6 \\
\hline
\end{tabular}

Tabla 3.- Valores de referencia recomendados (Norma UNI10829:1999).ӨT Rango aceptable de temperatura, $\Delta \theta T m a x$, variación máxima aceptable de temperatura; HRO, rango aceptable de humedad relativa; $\triangle H R$ max, variación máxima aceptable de humedad relativa; Norma UNI10829:1999 (Wills, et al., 2014). 


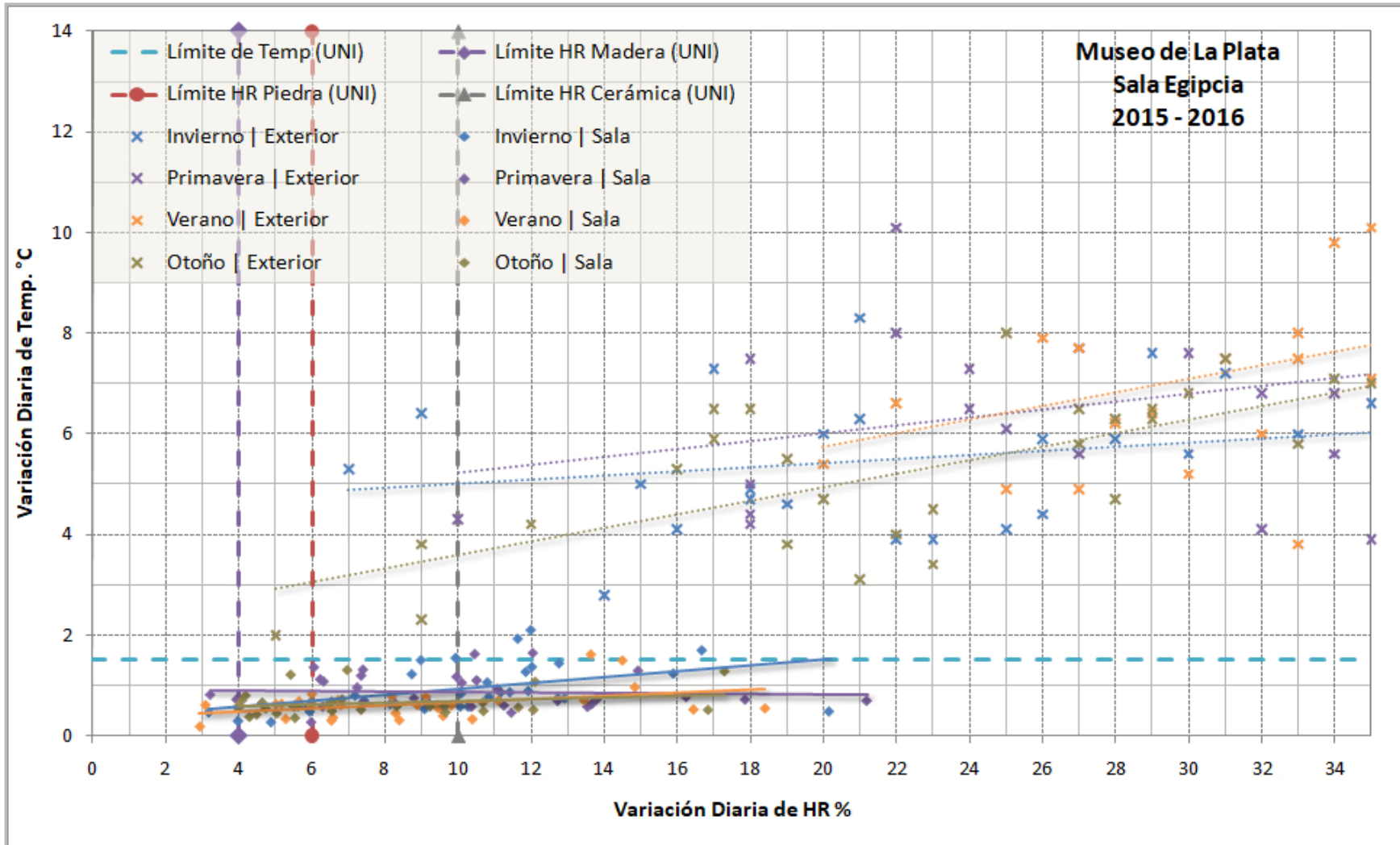

Figura 9- Variación diaria de temperatura y humedad en el exterior e interior de la Sala Egipcia durante las cuatro estaciones de muestreo: Límites recomendados de temperatura y humedad relativa para los distintos materiales. Puntos que marcan la variación diaria de T y HR en el interior de la sala; puntos que marcan la variación diaria de T y HR en el exterior del edificio.

\section{Discusión y conclusiones}

Durante el período total monitoreado se detectaron 18 tipos de géneros y especies fúngicas, siendo en su mayoría pertenecientes a anamorfos de Ascomycota (17) y solo uno con talo unicelular correspondiente a Basidiomycota. Estos resultados coinciden con los datos obtenidos por otros autores analizando contextos similares (Anaya et al. 2016; Borrego et al. 2010, 2012; Borrego \& Perdomo 2012, 2014, 2016; Borrego \& Molina 2014; Rojas et al. 2012). Con excepción de Acremonium, Alternaria, Fusarium solani, y Trichoderma, los restantes tipos fúngicos identificados se detectaron en las 8 campañas, aunque con distinta frecuencia. En el interior de la sala, Penicillium, Beauveria y Fusarium oxysporum fueron los tipos dominantes, mientras que en el exterior lo fue Cladosporium cladosporioides.

El análisis del índice RD estimado para los tipos fúngicos predominantes en todo el período en los 4 sitios indica que Aspergillus niger, Beauveria, Fusarium oxysporum y Penicillium fueron los tipos mejor representados.

Los resultados obtenidos aplicando el índice $1 / 0$ dan valores por debajo de 1,5 lo que indicaría que la ventilación está diluyendo los contaminantes internos o hay escasas fuentes de contaminación en el ambiente general, coincidiendo con estudios similares en sitios de guarda de bienes patrimoniales (Flores et al. 2014,
Kadaifciler 2017, Stryakowska-Sekuska et al. 2007). El análisis estadístico de las variaciones estacionales de las concentraciones fúngicas reveló diferencias significativas entre invierno/verano; primavera/verano; primavera/ otoño y otoño/ verano. Invierno fue la estación en que se registraron los valores más altos en los sitios 1,2 y 4 . Cuando se compararon los sitios entre sí, las diferencias en términos de concentración, no fueron significativas, con lo que se podría inferir que las variaciones dentro y fuera de la sala se mantienen constantes durante todo el año.

El análisis de la dinámica estacional de la biota fúngica muestra estabilidad cuali- cuantitativa entre los 3 sitios de la Sala y el exterior, considerando la calidad del aire exterior como un factor decisivo que influye en los niveles de contaminación en interiores (Kanaani et al. 2008; Lee 2006), además de los procesos de remoción e intercambio de aire propios de una Sala de exposición (Nayar \& Jothish 2013).

En coincidencia con nuestros estudios, la prevalencia de Aspergillus, Penicillium y Cladosporium ha sido frecuentemente registrada en estudios previos en otras salas de este Museo (Nitiu et al. 2015, 2016; Mallo et al, 2017) y en museos y archivos estudiados por otros autores (Anaya 2016; Čavka et al. 2010; Borrego \& Perdomo 2016; Flores etal.2014;Göksay-Kadaifciler \&Demirel 2017; Halem 
Khan \& Mohan Karuppayil 2012; Rojas et al. 2012; Shabir et al. 2007; Skóra et al. 2015; Unković et al. 2018). Este tipo de infecciones fúngicas en contextos de conservación, son causados por especies de lento crecimiento como los hongos mitospóricos xerofílicos pertenecientes a Ascomycota como Aspergillus, Paecylomyces, Penicillium y Cladosporium (Pinzari \&Montanari 2011; Sterflinger \& Piñar 2013). Además, es importante considerar que géneros con mayor representatividad en el espectro interior como Penicillium y Aspergillus desarrollan esporas que contienen proteínas relacionadas con la inmunoglobulina G (Ig G) y producen reacciones alérgicas (Knutsen et al. 2011; Shi \& Miller 2011). Asimismo, hongos muy presentes en el interior de la sala de los géneros Aspergillus, Fusarium y Penicillium son importantes debido a su capacidad de producción de micotoxinas.

La presencia del género Beauveria tiene una alta incidencia en la concentración total de la sala y reviste importancia debido a su notable habilidad proteolítica, en parte asociada a su papel como entomopatógeno (Saranraj \& Javaprakash 2017). Además, la disponibilidad de materiales con alto contenido proteico en momias y pergaminos, provee un sustrato rico en nitrógeno orgánico que podría promover la colonización por microorganismos y explicaría la carga de esporas de $B$. bassiana. Numerosos autores han destacado la importancia de muchos de los hongos identificados en este estudio como agentes de degradación de otros materiales debido a su variada actividad enzimática (Mallo et al. 2017; Robledo 1991; Ross \& Hollis 1976). Otro factor a tener en cuenta, se relaciona con el atractivo turístico de la sala que convoca a la mayoría de los visitantes, pudiendo ser éste también, un factor condicionante del ingreso de flujo contaminante. En referencia a la presencia de Rhodotorula, varios autores describen el aislamiento de este hongo de diferentes ecosistemas, incluidos los sitios con condiciones desfavorables. Previamente consideradas no patógenas, algunas especies de este género han emergido como patógenos oportunistas que tienen la capacidad de colonizar e infectar pacientes susceptibles (Wirth \& Goldani 2012). Su presencia en los 4 sitios, asociada a otros hongos proteolíticos presentes sugiere que la fuente principal procedería de materiales proteicos y derivados disponibles en los cuerpos momificados (Nitiu et al. 2018).

Para los ejemplares de las colecciones biológicas, los extremos de la temperatura y la humedad relativa en el ambiente de almacenamiento no son tan importantes al comparar estos valores con las fluctuaciones que pueden tener estos ambientes. Por lo tanto, se recomienda enfocarse en controlar especialmente las fluctuaciones de estas variables más que los valores extremos (Simmons \& Muñoz Saba 2005). Teniendo en cuenta el clima templado húmedo de la zona de La Plata y las características edilicias del Museo consideramos que es complejo alcanzar los valores óptimos a un costo energético y ambiental razonable, por este motivo cuando están disponibles se recomienda utilizar los valores de tolerancia.

Asimismo, el Cambio Climático ocasionado por la actividad antrópica se refleja tambien la inestabilidad de las variables ambientales que condicionan fuertemente la preservación del Patrimonio Cultural. Por ello, es prioritario realizar una evaluación continua de estos parámetros en Museos y Depósitos de resguardo para un manejo adecuado de estrategias de conservación.

El control de la temperatura y la humedad relativa (HR) simultáneamente con estrategias combinadas de monitoreo aerobiológico proporciona nuevas herramientas para la evaluación y predicción del tiempo de vida de los diferentes materiales y la estructura del edificio. Consideramos que para poder establecer una estrategia de control ambiental adecuada debemos evaluar los diversos factores que pueden afectar la conservación y cuidado de las colecciones, con el propósito de desarrollar las soluciones apropiadas.

\section{Agradecimientos}

Este estudio fue financiado por el Consejo Nacional de Investigaciones Científicas y Técnicas (CONICET) PIP 112201101-00087; Agencia Foncyt PICT 2013-0418, Agencia Foncyt PICT 2015-1620 y el Proyecto de Incentivos a la Investigación (N/897) de la Facultad de Ciencias Naturales y Museo (FCNyM), Argentina. Comisión de Investigaciones Científicas de la Provincia de Buenos Aires. Argentina. Se agradece el apoyo del Área de Conservación y Exhibición del Museo de Ciencias Naturales y Museo.

\section{Referencias}

ANAYA, M., BORREGO, S.F., GÁMEZ, E., CASTRO, M., MOLINA, A. \& VALDÉS, O. (2016). "Viable fungi in the air of indoor environments of the National Archive of the Republic of Cuba". Aerobiologia, 32, 513-527. https://doi.org/10.1007/s10453-016-9429-3

AVRAMI, E., DARDES, K., DE LA TORRE, M., HARRIS, S., HENRY, M., JESSUP, W. (1999). Evaluación para la Conservación: Modelo Propuesto para evaluar las Necesidades de Control del Entorno Museístico. Los Angeles: Getty Conservation Institute. http:// www.getty.edu/conservation/última consulta mayo 2019.

BARRON, G.L. (1968). The genera of Hyphomycetes from soil. Williams \& Wilkins, Baltimore. Pp 1-364

BAXTER A. (2011). On line document Air O Cell Interpretation guide (consulta enero 2019) Environmental Analysis Association.

BORREGO, S., GUIAMET, P., GÓMEZ DE SARAVIA, S., BATTISTONI, P., GARCIA, M., LAVIN, P. \& PERDOMO, I. (2010). "he quality of air at archives and the biodeterioration of photographs". International Biodeterioration and Biodegradation, 64(2), 139-145. https://doi. org/10.1016/j.ibiod.2009.12.005 
BORREGO, S., LAVIN, P., PERDOMO, I., GÓMEZ DE SARAVIA, S. \& GUIAMET, P. (2012). Determination of indoor air quality in archives and the biodeterioration of the documentary heritage. ISRN Microbiology, 680598. https://doi.org/10.5402/2012/680598.

BORREGO, S., PERDOMO, I. (2012). "Aerobiological investigations inside repositories of the National Archive of the Republic of Cuba". Aerobiologia, 28(3), 303-316. https://doi.org/10.1007/ s10453-011-9235-x

BORREGO, S. \& MOLINA, A. (2014). "Comportamiento de la aeromicrobiota en dos depósitos del Archivo Nacional de Cuba durante 7 años de estudio". AUGMDOMUS, 6, 1-24.

BORREGO, S.F. \& PERDOMO, I. (2014). “Caracterización de la micobiota aérea en dos depósitos del Archivo Nacional de la República de Cuba". Revista Iberoamericana de Micología, 31(3), 182-187. https://doi.org/10.1016/j.riam.2013.09.004

BORREGO, S. \& PERDOMO, I. (2016). Airborne microorganisms cultivable onnaturally ventilated documentrepositories of the National Archive of Cuba. Environ. Sci. Pollut. Res. 23: 3747-57. https://doi.org/10.1007/s11356-015-5585-1

CARMICHAEL, J. W, KENDRIK, W. B, CONNER, E. L, SIGLER, L. (1980). The genera of Hyphomycetes. Univ. Of Alberta Press, Edmonton. Pp 1- 386.

ČAVKA, M., GLASNOVIČ, A., JANKOVIČ, I., ŠIKANJIÉ, P. R., PERIÉ, B., BRKLJAČIÉ, B., MLINARIČ-MISSONI, E., ŠKRILIN, J. (2010). "Microbiological analysis of a mummy from the archaeological museum in Zagreb". Coll. Antropol. 34: 803 -805.

DOMCH, K. H., GAM, W., ANDERSON, T. H. (1980). Compendium of soil Fungi. Vol. 1, Academic Press, London. p. 860.

ELLIS, M. B. (1971). Dematiaceous Hyphomycetes. CMI Kew.

ESQUIVEL, P.P., MANGIATERRA, M, GIUSIANO, G, SOSA, M.A. (2003). "Microhongos anemófilos en ambientes abiertos de dos ciudades del nordeste argentino". Boletín Micológico 701: 21-8. https://doi.org/1022370/bolmicol.2003.18.0.376.

FLORES, M. E. B., MEDINA, P. G., CAMACHO, S.P. D., de JESÚS URIBE BELTRÁN, M., del CARMEN DE LA CRUZ OTERO, M. C.; RAMIREZ, I. O., HERNÁNDEZ, E. T.(2014) "Fungal spore concentration in indoor and outdoor air in university libraries, and their variations in response to changes in meteorological variables". Int J Environ Health Res. 2014 24(4):320-40. https://doi.org/10.1080/0960312 $\underline{3.2013 .835029}$

GARCÍA SANTA CRUZ, M. \& GÓMEZ, A. (2014) "Antecedentes y objetivos del Programa de Evaluación para la Conservación y Monitoreo Ambiental Edilicio". Actas del Encuentro Museos en Edificios Patrimoniales. Buenos Aires: CICOP Argentina, ICOM Argentina, Museo Banco Provincia de Buenos Aires.

GÖKSAY- KADAIFCILER, D. \& DEMIREL, R. (2017). “Fungal biodiversity and mycotoxigenic fungi in cooling-tower water systems in Istanbul", Turkey. J Water Health 15 (2): 308-320. https://doi.org/10.2166/wh.2017.274

GÓMEZ, A. (2009).Una aproximación al diseño ambientalmente consciente en espacios de guarda. Estudio de casos. Seminario de Investigación en Museología de los países de habla portuguesa y española. Porto: Universidade do Porto, Faculdade de Letras, Biblioteca Digital. ISBN: 978-972-8932-61-9.

HALEM KHAN, A.A. \& MOHAN KARUPPAYIL, S. (2012). "Fungal pollution of indoor environments and its management." Saudi Journal of Biological Sciences 19: 405-426. https://doi. org/10.2166/wh.2017.274

KADAIFCILER, D. G. (2017) "Bioaerosol assessment in the library of Istanbul University and fungal flora associated with paper deterioration". Aerobiologia33:151-166. https://doi.org/10.1007/ s10453-016-9457-z

KANAANI, H., HARGREAVES, M, RISTOVSKIA, Z, MORAWSKA, L. (2008) “Deposition rates of fungal spores in indoor environments, factors affecting them and comparison with non-biological aerosols". Atmospheric Environment 42(30):7141-7154. https:// doi.org/10.1016/j.atmosenv.2008.05.059

KNUTSEN, A.P, BUSH, R.K., DEMAIN, J.G., DENNING, D.W., DIXIT, A., FAIRS, A., GREENBERGER, P.A., KARIUKI, B., KITA, H., KURUP, VP., MOSS, R.B., NIVEN, R.M. PASHLEY,C.H., SLAVIN, R.G.,VIJAY, H. M., WARDLAW,A.J. (2011). "Fungi and allergic lower respiratory tract diseases". Clin. Rev. Alle. Immunol. 129: 280-91. 101016/j. jaci.2011.12970. https://doi.org/10.1016/j.jaci.2011.12.970

LEE, T., GRINSHPUN, S.A., MARTUZEVICIUS, D., ADHIKARI, A., CRAWFORD, C.M., REPONEN, T. (2006). "Culturability and concentration of indoor and outdoor airborne fungi in six single family homes". Atmospheric Environment 40: 2902-2910. https:// doi.org/10.1016/j.atmosenv.2006.01.011

LEVETIN E., SHAUGHNESSY R., FISHER E., LIGMAN B., HARRISON J., BRENNAN, T. (1995). “Indoor Air Quality in schools: exposure to fungal allergens". Aerobiologia 11:27-34. https://doi.org/10.1007/ BF02136141

MALLO, A.C., ELÍADES, L.A., NITIU, D.S., SAPARRAT, M.C.N. (2017). "Monitoring indoor air status in the Museo de La Plata Herbarium (LP) Argentina". Revista lberoamericana de Micología,34(2):99-105.

MALLO, A.C., NITIU, D.S., ELÍADES, L.A., SAPARRAT, M.C.N. (2019). Fungal degradation of cellulosic material used as support of Cultural Heritage. International Journal of Conservation Science IJS 8 (4)

MILLER, D.J., HAISLEY, P.D., REINHARDT, J.H. (2000). “Air sampling result in relation to extent of fungal colonization of building materials in some water-damaged buildings". Indoor Air, 10: 145151. https://doi.org/10.1034/j.1600-0668.2000.010003146.x

NAYAR, T.S., JOTHISH, P.S. (2012). “An assessment of the air quality in indoor and outdoor air with reference to fungal spores and 
pollen grains in four working environments in Kerala, India." Aerobiologia 29(1): 131 - 152. https://doi.org/10.1007/s10453012-9269-8.

NITIU, D.S., MALLO A.C., ELÍADES, L.A., SAPARRAT, M.C.N (2015). "Monitoreo de la carga fúngica ambiental y de otros bioaerosoles asociados a un depósito de restos momificados del NOA del Museo de la Plata (Argentina): un estudio de caso". Boletín de la Sociedad Argentina de Botánica 50 (4): 427- 436.

NITIU, D. S., MALLO A. C, ELÍADES, L. A., SAPARRAT. M .C. N, SANTA CRUZ, M. (2016). "Survey of the state of conservation of the Mylodon listai (XenarthraMylodontidae) skin fragment from the Pleistocene of Argentina kept at the Museum of La Plata (Argentina)". Ge conservación 10: 44 - 53. https://doi. org/10.37558/gec.v10i0.367

NITIU, D.S., MALLO, A.C., ELÍADES, L.A., GARCIA SANTA CRUZ, M., SAPARRAT, M.C.N. (2018). "Fungal monitoring in an exhibition room with Egyptian mummies in the Museum of Natural Sciences of La Plata, La Plata, Argentina". International Journal of Conservation Science (IJCS) 10 (2): 291-306.

NORMA UNI10586 (1997) Documentazione: Condizioni climatiche per ambienti di conservazione di documenti grafici e caratteristiche de glialloggiamenti. Milano Ente Nazionale di Unificazione

NORMA UNI10829 (1999) Beni di interessestorico e artistico: Condizioniambientali di conservazionemizurazione e danalisi. Milano Ente Nazionale di Unificazione

PINZARI, F. \& MONTANARI, M. (2011). "Mold growth on libraries material stored in compactus-type shelving units". En S. A. Abdul-Wahab Eds. Sick Building Syndrome:in Public Buildings and Workplaces.Pp 193 -206. Springer Berlag, Berlin Heidelberg. https://doi.org/10.1007/978-3-642-17919-8 11

PIÑAR, G., PIOMBINO- MASCALI, D., MAIXNER, F., ZINK, A., STERFLINGER, K. (2013). "Microbial survey of the mummies from Capuchin Catacombs of Palermo, Italy: biodeterioration risk and contamination of the indoor air"FEMS Microbiol Ecol. https://doi. org/10.1111/1574-6941.12165.

ROBLEDO, M.S.(1991) Caracterización taxonómica, distribución y algunos aspectos fisiológicos de Aspergillus spp. que deterioran documentos en el Archivo General de la Nación. Tesis de Maestría en Ciencias Biológicas. México. UNAM. (1991) http://oreon. dgbiblio.unam.mx.

ROJAS, T.I., AIRA, M.J., BATISTA, A., CRUZ, I.L., GONZÁLES, S. (2012). "Fungal biodeterioration in historic buildings of Havana, Cuba". Grana, 51(1), 44-51. https://doi.org/10.1080/00173134.20 $\underline{11.643920}$

ROSS, A.C. HOLLIS, B. "Microbiological deterioration of pulp wood, paper, and paint". In "Industrial Microbiology" (B. M. Miller and W. Litsky, eds.) (1976), pp 309-354. Mc. Graw-Hill, New York.
SARANRAJ, P., JAYAPRAKASH, A. BHAVANI, L. (2017)."Commercial production and application of bacterial alkaline protease: a review". Indo- Asian Multidisciplinary Research (IAJMR) 3 (5): 1228-1250. https://doi.org/10.22192/iajmr.2017.3.5.2.

SHABIR, A., KAHN, M. A., KAHAN, A.M., IQBAL; MAHMAD, F. (2007). "Fungal biodeterioration: a case of study in the Zoological Museum of Punjab University". J. Anim. PI. Sci 17 (3-4): 90 - 92.

SHI, C., MILLER, J. D. (2011) "Characterization of the 41 kDaallergenAspv 13, a subtilisin-like serine protease from Aspergillus versicolor". Mol. Immunol. 48:1827 -34.

SIMMONS, J. E., MUÑOZ SABA, Y. Eds. (2005). Cuidado, Manejo y Conservación de las Colecciones Biológicas. Conservación Internacional. Serie de Manuales para la conservación. Universidad Nacional de Colombia, Bogotá, Colombia.

SKÓRA, J., GUTAROWSKA, B., PIELECH-PRZYBYLSKA, K., STĘPIEŃ, Ł., PIETRZAK, K., PIOTROWSKA, M. \& PIETROWSKI, P. (2015). Assessment of microbiological contamination in the work environments of museums, archives and libraries. Aerobiologia, 31, 389-401. https://doi.org/10.1007/s10453-015-9372-8

SMITH, G. (1980). Ecology and field biology. Harper and Row. New York. $850 \mathrm{pp}$.

SØRENSEN, T. (1957). “A method of establishing groups of equal amplitude in plant sociology based on similarity of species and its application to analyses of the vegetation on Danish commons". Kongelige Danske Videnskabernes Selskab 5(4): 1-34.

STERFLINGER, K. \& PIÑAR, G. (2013). “Microbial deterioration of cultural heritage and works of art- tilting at windmills?" Appl. Microbiol. Biotechnol. 97: 9637 - 9046. https://doi.org/10.1007/ s00253-013-5283-1

STRYJAKOWSKA-SEKUSKA, M., PIOTRASZEWSKA-PAJAK, A., SZYSZKA, A., NOWICKI, M., FILIPAK, M. (2007). “Microbiological quality of indoor air in university rooms". Polish Journal of Environmental Studies. 16(4): 623-632.

UNKOVIĆ, N., DIMKIĆ, I., STANCOVIĆ, S., JELIKIĆ STANOJEVIĆ, D., POPOVIĆ, S., STUPAR, M., VUKOJEVIĆ, J., LJALJEVIĆ GRBIĆ, M. (2018) "Seasonal diversity of biodeteriogenic, pathogenic, and toxigenic constituents of airborne mycobiota in a sacral environment. Arh Hig Rada Toksikol; 69:317-327. https://doi. org/10.2478/aiht-2018-69-3194.

WILLS, B., WARD, C., SÁIZ GÓMEZ, V. (2014). "Conservation of Human Remains from Archeological Contexts" en Fletcher, A, Daniel, A., Hill, J. D Eds. Regarding the Dead: Human Remains in the British Museum. London: The British Museum.

WIRTH, F., GOLDANI, L. Z. (2012). “Epidemiology of Rhodotorula: an emerging pathogen". Interdiscip Perspect Infect Dis. 2012; 2012:465717. https://doi.org/10.1155/2012/465717 


\section{Autor/es}

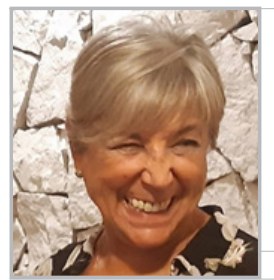

\section{Andrea Cecilia Mallo}

malloa2001@yahoo.com.ar

Facultad de Ciencias Naturales y Museo. Universidad Nacional de La Plata. Argentina.

Licenciada en Biología, orientación Botánica, Universidad Nacional de La Plata. Se desempeña como Personal de Apoyo a la Investigación, categoría Principal CIC - PBA en la temática de Aerobiología y Aeromicología desde el año 2006 en la Cátedra de Palinología de la Facultad de Ciencias Naturales y Museo. Docente en la Cátedra de Introducción a la Botánica en la Facultad de Cs Naturales y Museo, Categoría de Docente Investigador IV. Ha participado en 9 Proyectos de Investigación de la Universidad Nacional de la Plata y Comisión Nacional de Investigaciones Científicas y Técnicas (CONICET) y en la actualidad es integrante de Proyectos de Incentivos, UNLP; PIP CONICET,y ANPCyT. Ha sido Miembro de Comisiones Asesoras, Consejo Consultivo Departamental de Botánica de la Facultad de Ciencias Naturales y Museo (UNLP) evaluador de Proyectos de Investigación para la Agencia Nacional de Promoción Científica y Tecnológica (ANPCyT) y ha realizado arbitrajes en revistas nacionales e internacionales. Posee múltiples trabajos publicados en revistas científicas indexadas, capítulos de libro, trabajos completos en Actas de Congreso y presentaciones en congresos nacionales e internacionales. Ha realizado informes técnicos de distintas Salas del Museo de La Plata y del Archivo del Senado de la Provincia de Buenos Aires, Legislatura de la Provincia de Buenos Aires. Ha sido Codirectora de Pasantías y Becas en la Facultad de Ciencias Naturales y Museo.

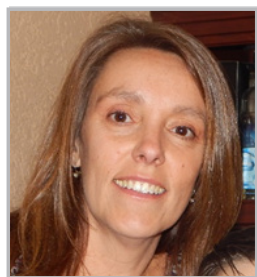

\section{Daniela Sivana Nitiu}

danielanitiu@yahoo.com.ar Facultad de Ciencias Naturales y Museo. Universidad Nacional de La Plata. Argentina.

Licenciada en Biología con Orientación Botánica y Doctora en Ciencias Naturales de la Universidad Nacional de La Plata. Investigadora del CONICET. Docente Investigador de la Cátedra de Palinología de la Facultad de Ciencias Naturales y Museo con Categoría de Docente Investigador II. Desarrolla investigación en el campo de la Aerobiología y la Aeromicología desde el año 1996. Directora de Proyectos de Investigación en la Universidad Nacional de La Plata. Evaluador de Proyectos de Investigación a nivel nacional e internacional. Ha realizado arbitrajes en revistas nacionales e internacionales. Directora de Tesis Doctoral (CONICET) y de pasantes para estudiantes de la FCNYM (UNLP). Posee numerosos antecedentes en Reuniones y Publicaciones en distintos ámbitos científicos nacionales e internacionales. Posee numerosos trabajos publicados en revistas científicas indexadas, capítulos de libro, trabajos completos en Actas de Congreso y presentaciones en congresos nacionales e internacionales. Ha recibido premios a trabajos científicos en diversos eventos científicos. Es miembro editor de la Revista Timisoara (Polonia). Es evaluador de diversas revistas científicas indexadas. Ha realizado Informes Técnicos para el Museo de La Plata y la Legislatura de la Provincia de Buenos Aires. Ha codirigido pasantías en la Facultad de Ciencias Naturales y Museo.

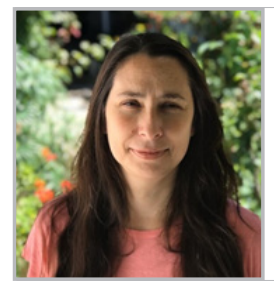

\section{Lorena Alejandra Elíades}

lorenaeliades@yahoo.com

Instituto Spegazzini . Facultad de Ciencias Naturales y Museo, Universidad Nacional de La Plata. Argentina

Licenciada en Biología orientación Ecología y Doctora en Ciencias Naturales, Títulos obtenidos en la Facultad de Cs Naturales y Museo, Universidad Nacional de La Plata (UNLP) Argentina. Se desempeña como Investigador Adjunto CONICET. Es Docente- Investigadora en la Cátedra de Botánica Sistemática I de la Facultad de Ciencias Naturales y Museo, con Categoría de Docente Investigador III. La labor desarrollada por la Dra Elíades se ha plasmado en 32 trabajos científicos de relevancia y dan cuenta de la diversidad de comunidades de hongos en el suelo, hongos como agente de deterioro de materiales de importancia histórica para la cultura humana y hongos como agentes solubilizadores de nutrientes conjuntamente con enzimas asociadas a la patogénesis de insectos. Desarrolla proyectos vinculados a diversidad y capacidades enzimáticas de interés biotecnológico, de hongos saprótrofos de suelos forestales y alcalinos. Ha presentado 37 trabajos en Congresos Nacionales, Latinoamericanos e internacionales. Ha concursado y obtenido diferentes subsidios para el financiamiento de su investigación en FONCyT Agencia de Promoción Cientíica y Tecnológica PICT y CONICET, PIP. Ha realizado análisis micológicos para diversas empresas y horticultores. Dirigió 6 estudiantes alumnos en pasantías entre 2014-1019. Es directora de dos tesistas doctorales de la Facultad de Ciencias Naturales y Museo de la UNLP. Se desempeña como evaluador de trabajos científicos de revistas internacionales y de resúmenes de presentaciones a congresos.
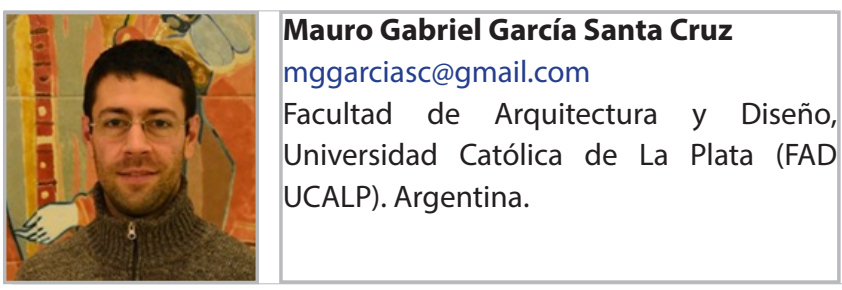

Arquitecto (FAU UNLP) y Especialista en Planeamiento Paisajista y Medio Ambiente, (FCAyF UNLP). Profesor adjunto de Arquitectura, Paisaje y Patrimonio (FAD UCALP), Investigador en Instituto de Investigación en Arquitectura y Territorio (INISAT FAD UCALP). Además es Investigador en Facultad de Bellas Artes, Universidad Nacional de La Plata (FBA UNLP) y Alumno del Doctorado en Arquitectura y Urbanismo (FAU UNLP). Obtiene Beca de Entrenamiento FAU UNLP (2007-2010) y Beca Doctoral CONICET (Abril 2014-Marzo 2019). Luego obtiene Beca Iberoamérica, Jóvenes Profesores e Investigadores, Santander Universidades (Abril - Junio 2019), FAD UCALP (Argentina) y PUC Minas (Brasil). Por último Beca a la Formación (2019), Fondo Nacional de las Artes (FNA Argentina). Desde 2007 participa en proyectos de investigación con financiamiento de ANPCyT, CONICET, 
UNLP y UCALP sobre arquitectura sustentable, conservación preventiva y gestión del patrimonio cultural. es autor y coautor de artículos publicados en revistas científicas con referato, capítulos de libros y trabajos publicados en las actas de eventos científicos. Actualmente es Miembro de International Council on Monuments and Sites (ICOMOS), International Scientific Committee on Energy, Sustainability and Climate Change (ICOMOS ISCES + CC), International Council of Museums (ICOM), International Committee for University Museums and Collections (UMAC ICOM), International Building Perfromance Simulation Association (IBPSA).

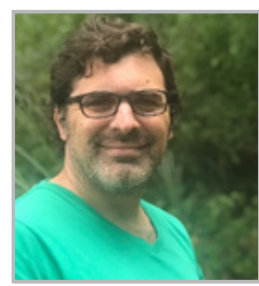

\section{Mario Carlos Nazareno Saparrat}

masaparrat@fcnym.unlp.edu.ar Facultad de Ciencias Naturales y Museo, Facultad de Ciencias Agrarias y Forestales, Universidad Nacional de La Plata. Argentina

Licenciado en Biología orientación Botánica. Doctor en Ciencias Naturales de la Universidad Nacional de La Plata. Investigador Independiente CONICET en el Instituto de Fisiología Vegetal de la Facultad de Ciencias Naturales y Museo. Profesor Titular UNLP en la Cátedra de Botánica Sistemática I (Facultad de Ciencias Naturales y Museo, UNLP) y Profesor Adjunto UNLP en la Cátedra Microbiología agrícola (Facultad de Ciencias Agrarias y Forestales, UNLP) y Vicepresidente de la Asociación Micológica Carlos Spegazzini. Desarrolla investigación en el campo de la fisiología de hongos y su rol en la transformación de materiales orgánicos y los pigmentos de los hongos, específicamente melaninas. Participa del Programa de Incentivos a los Docentes-Investigadores, Secretaría de Política Universitaria del Ministerio de Educación de la Nación en la Categoría II. Posee financiamiento para su investigación PICT como Investigador responsable (grupo en formación). Ha realizado diversas estancias y pasantías posdoctorales en el exterior. Ha recibido 4 distinciones. $\mathrm{Ha}$ sido docente expositor en numerosos cursos de postgrado. Ha sido coordinador y expositor en numerosos y diversos eventos científicos. Es director de varios becarios y tesistas en diferentes centros del país. Evaluador de Comisiones Científicas, Tesis y concursos docentes. Tiene en su haber numerosos arbitrajes de publicaciones científicas nacionales e internacionales y es Editor de sección sobre Plantas avasculares en la Revista Nacional "Darwiniana Nueva Serie". Ha participado en numerosos congresos y eventos científicos y presenta un alto número de publicaciones como primer autor, autor responsable (último) y en coautoría.

Artículo enviado el 10/07/2019 Artículo aceptado el 19/02/2020

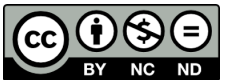

https://doi.org/10.37558/gec.v17i1.682 